\title{
Face Recognition using Eigenfaces
}

\author{
M A Imran \\ Computer Vision Lab \\ Department of Computer \\ Science \\ American International \\ University-Bangladesh
}

\author{
M S U Miah \\ Computer Vision Lab \\ Department of Computer \\ Science \\ American International \\ University-Bangladesh
}

\author{
H Rahman \\ Computer Vision Lab \\ Department of Computer \\ Science \\ American International \\ University-Bangladesh
}

\author{
A Bhowmik \\ Computer Vision Lab \\ Department of Computer Science \\ American International University-Bangladesh
}

\author{
D Karmaker \\ Computer Vision Lab \\ Department of Computer Science \\ American International University-Bangladesh
}

\begin{abstract}
We tried to develop a real time face detection and recognition system which uses an "appearance-based" approach. For detection purpose we used Viola Jones algorithm. To recognize face we worked with Eigen Faces which is a PCA based algorithm. In a real time to recognize a face we need a data training set. For data training set we took five images of each person and manipulated the Eigen values to match the known individual.
\end{abstract}

\section{Keywords}

Face Detection, Face Recognition, Eigen Faces, PCA

\section{INTRODUCTION}

Face recognition is becoming one of the major aspects in computer vision. Face Recognition have various applications including Security Systems, Augmented reality, Real time identification and many more. Over the past few decades there have been numerous works done in this field and many methods and algorithms have been proposed [1]. Although a revolutionary progress has been made regarding face recognition under usual circumstances and small variations but when it comes to recognizing faces in extreme variations like lighting and facial expressions the accuracy of the systems decreases dramatically. Fig. 1 shows how a same person under different light sources and different facial expressions appears to be different.
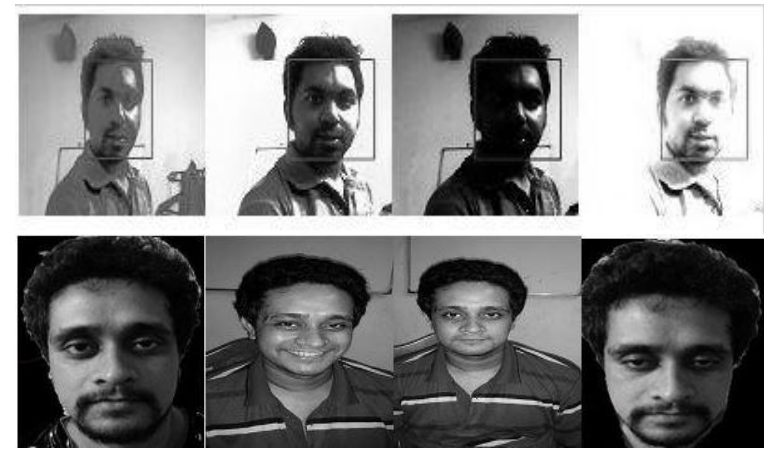

Fig 1: Different light and expression

In face recognition the main challenge arises with the variation in illumination, light sources, view angle, facial expressions [2] [3]. The difference between two images of the same person is induced due to illumination. This confuses the system based comparing images and they misclassify the identity of the input image. This phenomenon has been observed in [4] while using a data set of $n$ persons. Again we can see where different view angles may cause problems in fig.2. Here we can see how a same person viewed in different angles might appear to be different.

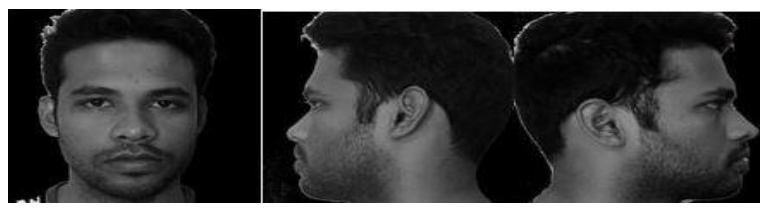

Fig 2: same person different angle

In our approach to face recognition we used Eigen Faces which is a PCA based algorithm. And to detect face we used 'Viola-Jones' algorithm. We took five images of each person and used them as a data set. Than we manipulated the Eigen values of the images to find a specific person.

\section{RELATED WORKS}

Experiment on automated human face recognition began in late 1960s .Bledsoe [5] developed a system that could automatically identify human facial features. First fully automated face recognition system was built by Kelly [6] and Kanade[7], it extract feature measurements from digital image and classily the feature vector. A verity of work had been done on neural networks [8], [9] to clear out the feature based methods. To build and match face models and facial features. Lapresté [10], Lee and Milios [11], Gordon [12] and others used range data. By the late 1980s, there had been a number of featured base facial recognition systems. But there were too many complications. Indexing schemes and other techniques were developed to cope up with these problems. In late 1987, as Sandy Pentland and Turk [13] began to work with appearance base facial recognition system. They started their work based on by Sirovich and Kirby [14] using Principal Components Analysis (PCA). In the mean while Burt [15] was developing a face recognition system by pyramids, multi resolution face representations. The era of appearance-based approach of face recognition was beginning with these works.

In spite of many problems there are a number of interesting features in Eigenface method. Since the past decade the amount of work done in this field has been increased dramatically. Since Turk and Pentland [16], Burt [15] and others began to work with appearance based methods for recognizing and detecting faces there has a tremendous amount of work on this topic. The Eigenface method threw away all the feature based information to test the limit of appearance based method for facial recognition. Moghaddam [17], Lanitis et al. [18] and others works on merging the two 
approaches. They predicted that this approach would be more accurate than the either approach alone. The Actual Eigenface framework did not considered the variation in light, facial expressions, viewing angle or any other factors that could change the facial image of an individual. In this case there would be a huge training set that would contain a large number of variations so that they could be modeled in Eigenfaces. Many works has been done for finding and characterizing for these variations [19] [20], the focus of these works was to merge the best qualities of feature-based and appearance-based approaches.

In terms of timing and impact some approaches are different from other. Craw [21] was one of the first who combines processing face shapes (2-D shapes) with eigenface-based recognition. To separate the photometric processing from geometric factors they normalize the face that images geometrically based on 34 face landmarks. Von der Malsburg and his colleagues [22], [23] invented a hybrid system based on elastic graph matching. This approach uses local grayscale information and combines it with global feature structure. Taylor and Cootes [24] created a combined approach for local and global information. To clearly model shape and intensity it uses flexible shapes.

In appearance-based facial recognition many tasks includes more sophisticated learning methods [25]. To use an extensive range of facial pose, warping and morphing face images [26] [27] are used. Independent Component Analysis (ICA) is an overview of PCA; it divides the high-order dependencies in the input, and also the second-order dependencies that PCA encodes [28]. A singular illustration and conversion for all facial images are used by the original eigenface method. No matter if they are created form one person or many; it also uses the easiest system possible. Moghad dam et al. [17] invented a probabilistic matching algorithm that separately model both interclass and intra class distributions using Bayesian approach. This algorithm improves the implicit assumption that images of all persons have a similar distribution. Penev and Sirovich [29] works with the dimensionality of face space; and they concluded that for a very large dataset, a minimum of 200 eigenfaces are required to adequately capture global variations such as pose difference ,race, sex, lighting etc. And for minor, identity-distinguishing details like nose, eye shape or exact eyebrow; at least twice many data set are required.

\section{EIGENFACES}

Eigenfaces are used in computer vision for human face recognition. Eigen faces assume ghastly appearance. Eigen faces refers to an appearance-based approach for face recognition. It captures the variation in the data set of face images which is latter used to convert and match images or individual persons. For distribution of faces Eigenfaces are the principle component. Eigenfaces is a crucial component for the performance of a facial recognition system.

Eigen Faces are used for: i) to get the appropriate facial info and ii) Efficiently produce facial image. Each of the images is represented with a minimum number of dimensions to reduce the space complexity and computation. To characterize the global variation with in the facial images, the Eigenfaces are used. Then the images that are connected with the largest Eigenvalues, they are estimated using a subset of the Eigenfaces. These subsets are responsible for most of the difference in the training set.

To get related information in facial image, we need to encode it efficiently and relate the face with a dataset of images encoded in the same way. To get the information contained in images one needs to obtain the variations in the data set, encode it independently and compare it with the individual face images.

Each of the specific faces can be characterized exactly according to linear combinations of Eigenfaces. Using specifically the "best" Eigenface, each face can estimate which has the biggest Eigenvalues and the dataset of the facial images.

Sirovich and Kirby [30] proposed the basic idea of using Eigenface. Using the PCA (principal component analysis), they started working with a collection of unique facial images and for image compression they calculated a best coordinate method. Here every coordinate is essentially an image which they named Eigen picture. They claimed that at least in theory, any set of facial image can be recreated by storing a lite set of weights for each of the faces and for the Eigen picture a small set is needed. These weights can be measured by projecting each of the images on to the Eigen picture. Using the characteristic feature, the magnitude of the facial images can be reconstructed [16]. To learn and recognize faces of the preferred personal, building up the characteristic features by practice is an efficient way. So each person would be considered by a small set of features or the weights of the Eigen picture needed to be reconstructed. This is a very solid representation of the images compared to the dataset.

\subsection{Summary of the Face Recognition Process}

-Initialization step:

1. Take a primary set of face images, whose pixels are same $(\mathrm{N} \times \mathrm{N})$ and each person has 5 sample. It's called training set.

2. From the training set calculate the Eigen faces, keeping only the $\mathrm{M}$ images corresponding to the highest Eigen values. Face spaces are defined by these $\mathrm{M}$ images.

3. The distribution in the images (M dimensional weight space) needs to be calculated for each known persons, and their face images needs to be projected on to the face space.

-After initializing the system, these following steps are used for recognizing new faces:

4. A set of weight depending on the input image needs to be calculated and on each of the Eigen Faces the $\mathrm{M}$ Eigen faces projects the input image

5. If an image is a face or not that needs to be determine by checking if the image is adequately close to face to face.

\subsection{Training}

\subsubsection{Step 1:}

The training images $\left[I_{1,1 \ldots 1,5}, I_{2.1 \ldots 2.5}, \ldots I_{M .1 \ldots . . . .5}\right]$ of dimensions $\mathrm{N} * \mathrm{~N}$ are analyzed. $I_{1,1 \ldots 1,5} \mathrm{ss}$ the sample image of $1^{\text {st }}$ person. Same as $I_{2,1 \ldots 2,5}$ are $2^{\text {nd }}$ person's sample face and so on. A training set of $N^{2} * 5 * \mathrm{~m}$ dimensions is thus created, where $m$ is the number of sample images. Figure 3 shows the 5 sample per person.

3.2.2 Step 2:

Collect all gray levels in a long vector $\mathrm{u}$

$$
u=\left(I_{1,1 \ldots 1.5} I_{2,1 \ldots 2 \ldots .5} I_{M, 1 \ldots M .5}\right)^{T}
$$




\subsubsection{Step 3:}

Collect $\mathrm{n}$ samples (views) of each of $\mathrm{p}$ persons in matrix A

(NN X pn): $\mathrm{A}=\left[u_{1}^{1} \ldots u_{n}^{1}, u_{1}^{2} \ldots u_{n}^{2}, \ldots u_{1}^{p} \ldots u_{n}^{p}\right]$

\subsubsection{Step 4:}

Compute eigenvectors of C.

\subsubsection{Step 5:}

Compute eigenvectors of $\mathrm{L}$ from eigenvectors of $\mathrm{C}$.

\subsubsection{Step 6:}

Select few most significant eigenvectors of $L$ for face recognition.

\subsubsection{Step 7:}

Compute coefficient vectors corresponding to each training image.

\subsubsection{Step 8}

For each person, coefficients will form a cluster

\subsection{Recognition}

\subsubsection{Step 1:}

Create a vector $\mathrm{u}$ for the image to be recognized.

\subsubsection{Step 2:}

$$
u=\left(I_{1,1 \ldots 1.5}\right)^{T}
$$

Compute coefficient vector for this $\mathrm{u}$.

\subsubsection{Step 3:}

Decide which person this image belongs to, based on the distance from the cluster mean for each person.

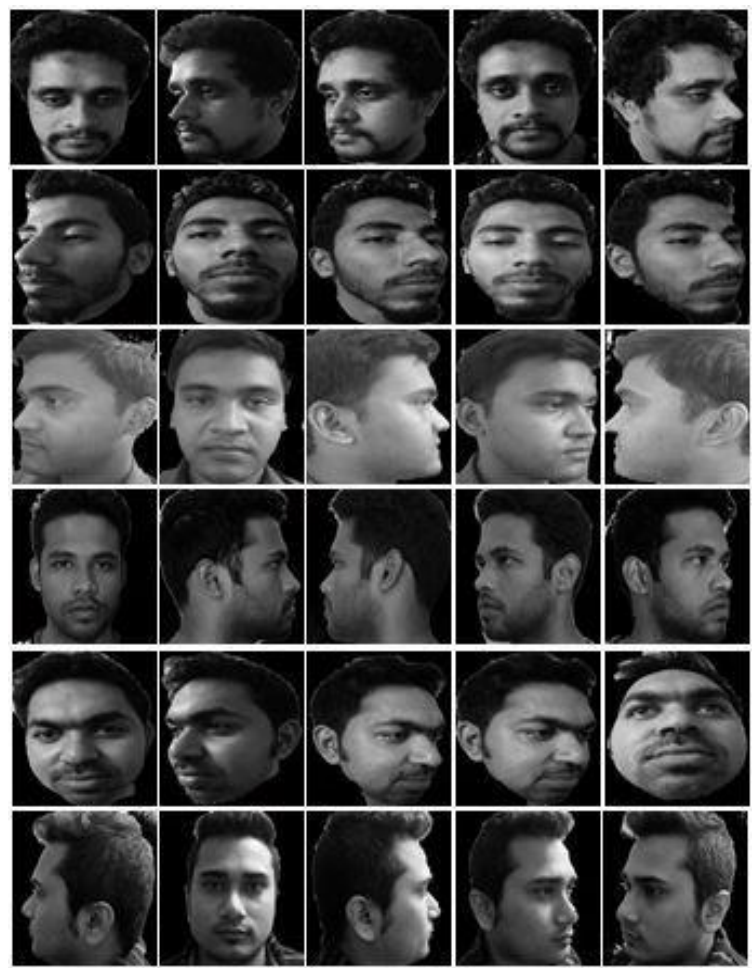

Fig 3: each person has 5 samples

\section{FACE DETECTION}

We used Viola-Jones algorithm for face detection. The System object has some pre-trained classifiers that can detect some facial features [33] line profile face, front face, nose, eyes, ear etc. But due to many limitations in face detection technology like illumination, pose etc. these classifiers can be customized. These custom classifiers can be trained by trainCascadeObjectDetector function which is provided by Computer Vision System Toolbox ${ }^{\mathrm{TM}}$

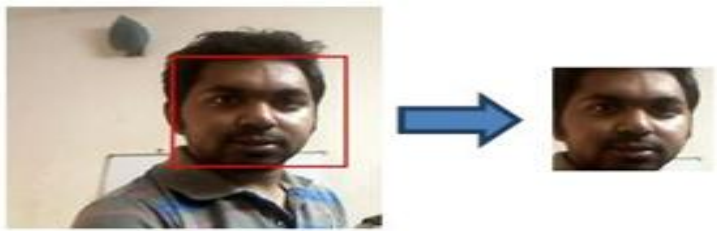

Fig 4: mug shot generation

\section{ALGORITHM}

$o b j=$ VideoDevice

$i m g$ CaptureNextFrame $(o b j)$

FaceDetect $=$ DetectFaceInMotion

$B B=$ CaptureNextFrame (FaceDetect, img)

FOR $i=1$ TO size of BB DO

CREATE rectangle(parameters);

ENDFOR

frame=ImageResize(frame, resolution);

FOR $i=1$ TO size of $B B D O$

frame $=$ CropImage $($ image, parameters $)$

ENDFOR

CREATE imagefiles (path to file)

NumberofFiles = COUNT imagefiles

FOR $i=1$ TO NumberOfFiles DO

FOR $j=1$ TO $(i+4)$ DO

CurrentFile = ReadALL (path_of_file_j)

CurrentImage $=$ READ CurrentFile

CurrentImage = ConvertToGray (CurrentImage)

$U=$ Reshape (CurrentImage)

$U=\operatorname{Inverseof}(U)$

IF $j=1$ THEN

$A=U$

ELSE

$A=A X U$;

ENDFOR

ENDIF

$$
\text { ENDFOR }
$$

invA $=$ Inverseof $(A)$

$C=$ invA $\times A$

$\operatorname{arr}[$ vec, val $]=\operatorname{Eigenof}(C)$

ss $=$ Diagonal (val)

$\operatorname{arr}[s s, i g]=$ DescendingSort(ss)

vec $=$ vec $X i g$

$\operatorname{vec} L=A X$ vec

coeff $=$ invA $X$ vecL

totalPerson $=$ NumberofFiles $/ N$

FOR $i=1$ TO totalPerson DO

Model $(i)=\operatorname{Mean}\left(\operatorname{coeff}\left(N^{*} 1\right.\right.$ TO $\left.\left.N^{*}(i-1)+1\right)\right)$

END FOR

WHILE(TRUE)

CurrentImage $=$ ConvertToGray (frame)

$U=$ Reshape(CurrentImage)

imgco $=U X$ vecL

top $=1$

FOR $i=2$ TO totalPerson

IF(Normalize(Model (i)) -imgco <

Normalize(Model(top))-imgco) THEN UPDATE top

ENDFOR

$$
\text { ENDIF }
$$

\section{ENDWHILE}

\section{PROBLEMS \& SOLUTIONS}

This algorithm chooses the closest person when recognizing an unknown face image. There is no threshold value in this algorithm. We need to choose a threshold value depending on the environment and whose value is greater than Euclidian distance.

if $e_{r}<T_{r}$, then $\Gamma$ is recognized as face 1(which would be black) from the training set

Here $\quad e_{r}=$ distance within the face space

$T_{r}=$ threshold value. 
This method is really sensitive to scale. So a low level processing is essential for scale normalization.

For different poses and illumination, the recognition rate decreases. The Fisherface projection approach can solve the problems of illumination. It solves the problem by maximizing the ratio between class scatter to within-class scatter. Never the less a projection that is able to instantaneously differentiate numerous face classes is almost impossible.

When dealing with expression and glasses Eigenface approach can robust. Most of these experiments were done only with frontal view. It can be more problematic when there are extreme changes [34] in the pose, expression or the person is in disguise.

The dataset containing the face images that are used for the experiments are taken in a default background. But the background can change in the real world scenarios; this will hamper the recognition performance. So for real life uses segmentation process needs to be considered.

Additionally, due to Eigenface algorithms "appearance-based" features, the face recognition process has some common disadvantages. First, training the data set is a lengthy process. So it's really time consuming to update the database. Second, when the dimensions of the face space is smaller than the number of face classes only that time the recognition system is efficient.

For change in lighting, distance and angel there is partial robustness [33]. And $2 \mathrm{~d}$ recognition system do not capture the real size of the face. This creates some fundamental problem [32].

\section{PROPOSED METHOD}

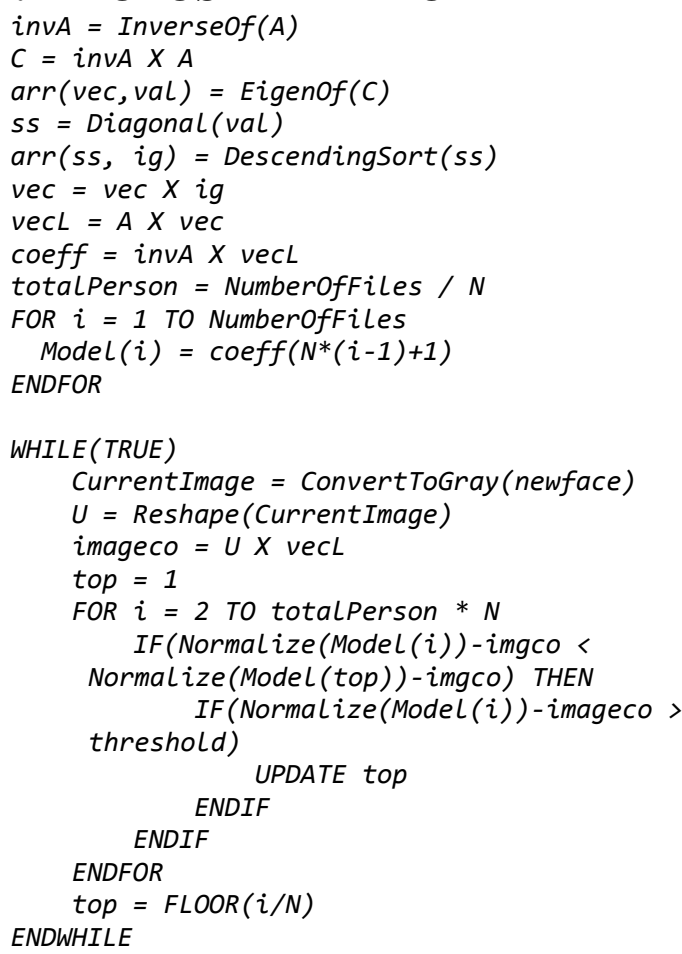

For the previous method, if a person's face isn't in the dataset, it will still select the image is known as the training image with which its score is the lowest. This is a problem that we need to look at. For this purpose we decide the threshold value. The threshold value is chosen arbitrarily. There is no formula to calculate the threshold value. Its value is chosen arbitrarily or obtained as some factor of maximum value of the minimum Euclidian distances of every single image from other images. Another feature is 1st person image (10 sample) will be black so if no match person is found we can select it as unknown person. The proposed algorithm works with 10 sample of per person and gets the image coefficient value and compares with the new face image's coefficient value. This may more accurate. Below is the algorithm we proposed.

\section{COMPARISON WITH EXISTING ALGORITHM}

For the previous algorithm accuracy was $45 \%$. For the proposed algorithm accuracy is $80 \%$. Proposed algorithm will work each of the sample image (not average value) and store data which will compare the new face image coefficient. The proposed algorithm will more accurate when dataset is over 2000.
For Previous algorithm

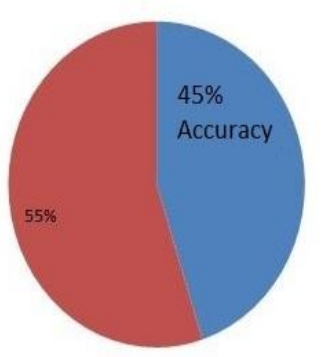

For Proposed algorithm

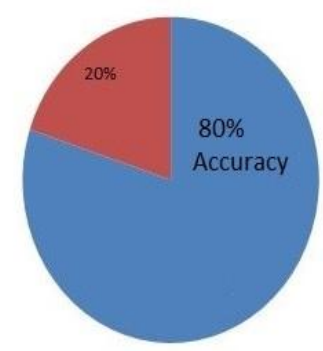

Fig 5: Accuracy measurement

\section{CONCLUSIONS}

The most challenging part for face recognition using Eigenface is the changes in the details like, background, illumination, facial expression, pose, scale sensitivity etc. These problems decrease the efficiency of the system. These problems can be somewhat manageable but not totally avoidable. Though a tremendous amount of research has been done in this field, upgrades can be done to the PCA based Eigenface method.

\section{REFERENCES}

[1] A. Samal and P. Iyengar, "Automatic Recognition and Analysis of Human Faces and Facial Expressions: A Survey," Pattern Recognition,vol. 25, pp. 65-77, 1992.

[2] WenYi Zhao, Rama Chellappa "Image based Face Recognition Issues and Methods" urlhttp://www.pbs.org/wgbh/nova/next/tech/the-limits-offacial-recognition/

[3] Y. Adini. Y. Moses and S. Ullman, "Face Recognition: The problem of Compensating for Changes in Illumination Direction," IEEE Trans. on PAMI, Vol. 19, pp.721-732, 1997

[4] W.W. Bledsoe, "Man-machine facial recognition," Technical Report PRI 22, Panoramic Research Inc., Palo Alto, CA, Aug. 1966.

[5] M.D. Kelly, "Visual identification of people by computer," Stanford Artificial Intelligence Project Memo AI-130, July 1970. 
[6] T. Kanade, "Picture processing system by computer complex and recognition of human faces," Dept. Information Science, Kyoto University, Nov. 1973.

[7] D. Valentin, H. Abdi, A.J. O'Toole, and G.W. Cottrell, "Connectionist models of face processing: A survey," Pattern Recognition, vol.27, pp.1209-1230, 1994.

[8] M. Flemming and G. Cottrell, "Face recognition using unsupervised feature extraction," Proc. Intl. Neural Network Conf., Paris, 1990.

[9] J.T. Lapresté, J.Y. Cartoux, and M. Richetin, "Face recognition from range data by structural analysis," in Syntactic and Structural Pattern Recognition, ed. G. Ferrat, et al., NATO ASI series, vol.F45, SpringerVerlag, Berlin, Heidelberg ,1988.

[10] J.C. Lee and E. Milios, "Matching range images of human faces," Proc. IEEE Third Intl. Conf. Computer Vision, pp.722-726, Osaka, Japan, Dec. 1990.

[11] G.G. Gordon, "Face recognition from depth and curvature," Ph.D. Thesis, Harvard University, 1991.

[12] Matthew TURK ,"A Random Walk through Eigen space," IEICE TRANS. INF. \& SYST., VOL.E84-D, NO.12 DECEMBER 2001

[13] L. Sirovich and M. Kirby, "Low dimensional procedure for the characterization of human faces," J. Optical Society of America, vol.4, no.3, pp.519-524, 1987.

[14] P. Burt, "Smart sensing within a pyramid vision machine," Proc. IEEE, vol.76, no.8, pp.1006-1015, 1988.

[15] M. Turk and A.P. Pentland, "Eigenfaces for recognition," J. Cognitive Neuroscience, vol.3, no.1, pp.71-96, 1991.

[16] B. Moghaddam, W. Wahid, and A. Pentland, "Beyond eigenfaces: Probabilistic matching for face recognition," Proc. Third Intl. Conf. Automatic Face- and Gesture Recognition, pp.30-35, Nara, Japan, 1998.

[17] A. Lanitis, C.J. Taylor, and T.F. Cootes, "Automatic interpretation and coding of face images using flexible models," IEEE Trans. Pattern Anal. \& Mach. Intell., vol.19, no.7 pp.743-756, 1997.

[18] A.S. Georghiades, D.J. Kriegman, and P.N. Belhumeur, "Illumination cones for recognition under variable lighting: Faces," IEEE Conf. Computer Vision and Pattern Recognition, 1998.

[19] L. Zhao and Y.H. Yang,"Theoretical analysis of illumination in PCA-based vision systems," Pattern Recognition, vol.32, pp.547-564, 1999.

[20] I. Craw, N. Costen, T. Kato, G. Robertson, and S. Akamatsu, "Automatic face recognition: Combining configuration and texture," Proc. Intl. Workshop on Automatic Face and Gesture-Recognition, pp.53-58, Zurich, 1995.

[21] M. Lades, J.C. Vorbruggen, J. Buhmann, J. Lange, C. Von der Malsburg, R.P. Wurtz, and W. Konen, "Distortion invariant object recognition in the dynamic link architecture," IEEE Trans. Comput., vol.42, no.3, pp.300-311, 1993.
[22] R.P. Würtz, J.C. Vorbrüggen, C. von der Malsburg, and J. Lange, "Recognition of human faces by a neuronal graph matching process," in Applications of Neural Networks, ed. H.G. Schuster, pp.181-200, VCH, Weinheim, 1992.

[23] A. Lanitis, C.J. Taylor, and T.F. Cootes, "A unified approach to coding and interpreting faces," Proc. 5th Intl. Conf. Computer Vision, pp.368-373, 1995.

[24] Y. Li, S. Gong, and H. Liddell, "Support vector regression and classification based multi-viewface detection and recognition," Proc. Conf. Automatic Face and Gesture Recognition, pp.300-305, Grenoble, France, March 2000.

[25] T. Ezzat and T. Poggio, "Facial analysis and synthesis using image-based models," Proc. Second Intl. Conf. Automatic Face and Gesture Recognition, pp.116-121, Killington, VT, 1996.

[26] M. Bichsel, "Automatic interpolation and recognition of face images by morphing," Proc. Second Intl. Conf. Automatic Face and Gesture Recognition, pp.128-135, Killington, VT, 1996.

[27] T.W. Lee, Independent Component Analysis: Theory and Applications, Kluwer Academic Publishers, Dordrecht, 1998.

[28] P.S. Penev and L. Sirovich, "The global dimensionality of face space," Proc. 4th Int'l. Conf. Automatic Face and Gesture Recognition, pp.264-270, Grenoble, France, 2000 .

[29] Kirby, M. and L. Sirovich, Application of the KarhunenLoève Procedure for the Characterization of Human Faces. IEEE Transactions on Pattern Analysis and achineIntelligence, 1990. 12(1): p. 103-108

[30] Saha and Bhattacharjee "Face Recognition Using Eigen faces", (ISSN 2250-2459, ISO 9001:2008 Certified Journal, Volume 3, Issue 5.2013

[31] Trina D. Russ, Mark W. Koch, Charles Q. Little, "3D Facial Recognition: A Quantitative Analysis," 38th Annual 2004 International Carnahan Conference on Security Technology, 2004.

[32] John D. Woodward, Jr., Christopher Horn, Julius Gatune, Aryn Thomas, "Biometrics, A Look at Facial Recognition," RAND, 2003.

[33] M A U Rahman, M S U Miah, M A Fahad and D Karmaker, "SHIMPG: Simple human interaction with machine using Physical Gesture" Control Automation Robotics \& Vision (ICARCV), 2014 IEEE. [Online]. Available:

http://ieeexplore.ieee.org/xpl/articleDetails.jsp?arnumber $=7064322$.

[34] D karmaker, H Rahman, M S Rahaman, M S Rahman, "Global Motion tracking with six parameter model", ARPN Journal of Systems and Software,Volume 1 No. 5 , 2011. [Online]. Available: http://scientificjournals.org/journalofsystemsandsoftware/archive/vol1n o5/vol1no5_3.pdf. 